\title{
Impact of Electronic Cigarettes on the Liver
}

\author{
Alaa Ramadan ${ }^{1}$, Mohamed Diaa ${ }^{2}$ and Khaled A Abdel-Sater ${ }^{3 *}$ \\ ${ }^{1,2}$ Student, Qena Faculty of Medicine, Egypt \\ ${ }^{3}$ Department of Physiology, Egypt
}

ISSN: 2640-9208

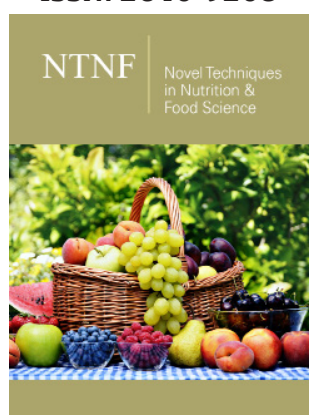

*Corresponding author: Khaled A Abdel Sater, Department of Physiology, Egypt

Submission: 制September 13, 2021

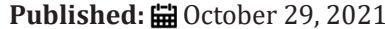

Volume 6 - Issue 2

How to cite this article: Alaa Ramadan, Mohamed Diaa, Khaled A Abdel-Sater. Impact of Electronic Cigarettes on the Liver. Nov Tech Nutri Food Sci. 6(2). NTNF. 000633. 2021. DOI: 10.31031/NTNF.2021.06.000633

Copyright@ Khaled A Abdel-Sater. This article is distributed under the terms of the Creative Commons Attribution 4.0 International License, which permits unrestricted use and redistribution provided that the original author and source are credited.

\section{Abstract}

E-cigarettes can affect several organs in the body. On the liver, it causes toxic and immunological effects associated with many inflammatory processes and oxidative stress. E-cigarettes may cause hepatic fibrosis, steatosis, cell dysfunction, injury with the elevation of liver enzymes and cancer. On the other hand, it also causes hepatic DNA damage and mitochondrial dysfunction. Nicotine affects both cellular and humoral immune responses.

Keywords: ECs; E-cigarettes; Nicotine; Propylene glycol; Glycerol; Liver

\section{Introduction}

E-cigarettes, also known as electronic cigarettes, electronic vaping devices electronic nicotine delivery systems or ECs, are battery-operated devices with the function to vaporize nicotine ${ }^{1}$. There are several types of ECs: more than 460 e-cigarette brands and 8000 different flavorings. The products available on the market are mainly of three types: first, second, and third generations ${ }^{2}$. First-generation devices have a similar appearance, shape and size as tobacco cigarettes. These were the first ECs released to the market. They comprise of a little lithium battery and a cartomizer. Examples of the first generation are cigalikes, vape sticks and vape pen [1-8]. Second-generation devices are characterized by higher-capacity lithium batteries and atomizers with the ability to refill them with liquid (sold in separate bottles). Third-generation devices are composed of a lithium battery with an integrated circuit that allows the user to adjust the energy delivered to the atomizer. They are also called mods. Box Mod is a crate formed e-cigarette, named after its shape like a case. Mech Mod is a mechanical smoke, an e-cigarette gadget that doesn't contain a control chip, and its security relies upon the information on the player. Pod Mod is a shut e-cigarette with replaceable cartridges. HnB is a heat-not-burn smoking device and E-hookah is an electronic hookah ${ }^{3}$. Most variety of ECs comes from the different nicotine present in e-liquids, different volumes of e-liquids per product, different carrier compounds, additives, flavors, and battery voltage ${ }^{4}$.

Components identified in e-cigarette liquids and aerosols include nicotine, humectants (propylene glycol and glycerol), polycyclic aromatic hydrocarbons, tobacco alkaloids, tobaccospecific nitrosamines, phenolic compounds, water, aldehydes, citric acid, heavy metals,

${ }^{1}$ Gurjot K, Muthumalage T, Rahman I (2018) Mechanisms of toxicity and biomarkers of flavoring and flavor enhancing chemicals in emerging tobacco and non-tobacco products. Toxicology Letters 288: 143-155.

${ }^{2}$ (2018) Committee on the Review of the Health Effects Systems, Electronic Nicotine Delivery, National Academies of Sciences, Engineering, and Medicine; Health and Medicine Division; Board on Population Health and Public Health Practice. In: Eaton DL, Kwan LY, Stratton K (Eds.), (13 ${ }^{\text {th }}$ edn), Washington-United States of America: Public Health Consequences of E-Cigarettes. National Academies Press, USA.

${ }^{3}$ Cao Y, Wu D, Ma Y, Ma X, Wang S, Li F, et al. (2021) Toxicity of electronic cigarettes: a general review of the origins, health hazards, and toxicity mechanisms. Science of the Total Environment 772: 145475.

${ }^{4}$ Grana R, Benowitz N, Glantz SA (2014) E-cigarettes a scientific review. Circulation 129(19): 1972-1986. 
flavoring agents, and volatile organic compounds ${ }^{5}$. Users of ECs often report that propylene glycol produces better "throat hit" and carries flavor better than glycerol while glycerol is much smoother than propylene glycol [8-14]. Propylene glycol is physically much thinner than glycerol. Some of the key metals include iron, nickel, lead, tin, aluminum, manganese and chromium. All metals except cadmium, are found at a markedly higher level than in combustible tobacco cigarettes ${ }^{6}$.

Heating this liquid produces an aerosol (e-vapor), which is toxic, oxidative, and induces inflammation ${ }^{7}$. Nicotine causes dependence and addiction, and exposure to it from ECs likely elevates the cardiovascular disease risk in people with pre-existing cardiovascular diseases, but the cardiovascular risk in people without cardiovascular diseases is uncertain ${ }^{8}$. There are more than 8000 different flavoring agents (e.g. menthol, fruit, vanilla, caramel, and coffee) of e-liquid available. Some flavorings resemble the taste of cigarettes ${ }^{9}$. The majority of them are assigned as commonly perceived as safe by FDA, however, those assignments are for oral utilization in food and do not apply to flavorings used in ECs; most of these were never studied for toxicity via the inhalation route ${ }^{10}$.

By the end of 2021, the number of e-cigarette users- may reach almost 55 million worldwide ${ }^{11}$. Youths ages 15 to 25 years had the highest rate of using e-cigarette ${ }^{12} .5 .3 \%$ of all users are middle school students, and $16 \%$ are high school students. This is very dangerous because the brain is only fully developed by the age of mid-twenties, youths' exposure to nicotine may disrupt their brain development, and hinder attention and learning while elevating susceptibility for addiction to nicotine or other drugs such as cocaine $^{13}$. The use of ECs among the young is essentially connected to their curiosity and the "appealing" flavored nature of e-liquids [15-20]. Former smokers primarily use ECs to discontinue smoking because they perceive them to be safety perceptions, current trends, and reduced costs compared with tobacco cigarette smoking ${ }^{14}$. Users believe that the negative effects of ECs use can only become apparent after many years. However, there are certain differences of opinion about its long-term effects on health. In particular, some people assert that it is less harmful than traditional cigarettes, but others assert the opposite ${ }^{15}$.

Users claim safety aid because ECs purportedly do not involve tobacco combustion, which reduces toxicant exposure for e-cigarette users as compared to traditional cigarettes. But these data are not conclusive. ECs may provide a less harmful source of nicotine than traditional cigarettes, but evidence of decreased harm with long-term use is not available. Some proof suggests that e-cigarette use may facilitate smoking cessation, but definitive data are lacking. No e-cigarette has been approved by FDA as a cessation aid $^{16}$. The use of ECs mainly affects the respiratory tract, cardiovascular system, central nervous system, reproductive system, immune system, mouth, eye, nose, throat and skin. Also, ECs can damage the digestive system. On the oral cavity, ECs cause oral tissue dehydration, a decrease of the flow of gingival fluid, a decrease of enamel hardness, a decrease of lysozyme levels in saliva and elevation levels of lactoferrin in saliva [21]. ECs also cause reflux esophagitis and cancer of the esophagus. On the other hand, ECs also destroy insulin-secreting cells which lead to diabetes mellitus ${ }^{17}$.

Because of these contemplations, it is clear that there are many unanswered questions regarding the overall safety, efficacy of harm reduction, and the long-term health impact of these devices. This article aims to give a critical review of the existing studies on the health consequences of vaping of ECs on the liver.

${ }^{5}$ Mikheev VB, Brinkman MC, Granville CA, Gordon SM, Clark PI (2016) Real-time measurement of electronic cigarette aerosol size distribution and metals content analysis. 1895-1902.

${ }^{6}$ Committee on the Review of the Health Effects Systems.

${ }^{7}$ Worsley DJ, Jones K, Marshman Z (2014) Patients are asking about e-cigarettes. what do we tell them? British Dental Journal 217(2): 91-95.

${ }^{8}(2010)$ U.S. Department of Health and Human Services, How Tobacco Smoke Causes Disease: The Biology and Behavioral Basis for Smoking-Attributable Disease, How Tobacco Smoke Causes Disease: The Biology and Behavioral Basis for Smoking-Attributable Disease: A Report of the Surgeon General.

${ }^{9}$ Kaur, Muthumalage, and Rahman.

${ }^{10}$ Committee on the Review of the Health Effects Systems.

${ }^{11}$ Kaur, Muthumalage, and Rahman.

${ }^{12}$ Cao and others.

${ }^{13}$ Hanan Q, Karim ZA, Rivera JO, Khasawneh FT, Alshbool FZ (2017) Impact of electronic cigarettes on the cardiovascular system. Journal of the American Heart Association 6(9): e006353.

${ }^{14}$ Gerard Li, Saad S, Oliver BG, Chen H (2018) Heat or burn? impacts of intrauterine tobacco smoke and e-cigarette vapor exposure on the offspring's health outcome. Toxics 6(3): 43.

${ }^{15}$ Majeed BA, Dube SR, Sterling K, Whitney C, Eriksen MP (2015) Opinions about electronic cigarette use in smoke-free areas among U.S. adults, 2012. Nicotine and Tobacco Research 17(6): 675-681.

${ }^{16}$ Lyon PC (2014) Electronic cigarettes: human health effects. Tobacco Control 23(Suppl 2).

${ }^{17}$ Cao and others. 


\section{The Hepatic Effects of ECs}

The liver is a significant organ that has numerous functions. The liver is responsible for removing drugs, alcohol and other toxins from the body. Also, it is for nicotine transformation, and nicotine exerts several of adverse physiological effects on the liver ${ }^{18}$. Various chemical substances and ultrafine particles known to be toxic, carcinogenic, and/or to cause liver disease to have been identified in e-cigarette aerosols, cartridges, refill liquids, and environmental emissions. The toxicity of ECs is not only attributed to nicotine but also by aldehydes, metals, volatile organic compounds, phenolic compounds, polycyclic aromatic hydrocarbons, and tobacco alkaloids and flavoring agents' mixture in e-liquid ${ }^{19}$. Indeed, exposure to humectants aerosols in concentrations found in ECs has no hepatic effect but several hazardous compounds have been found in liquids and in the heated aerosol produced by ECs, including formaldehyde, acetaldehyde, and acrolein, which are known carcinogenic toxicants ${ }^{20}$.

The exposure to ECs chemical substances is highly variable and depends on product characteristics and how the device is operated. Nicotine, 3-(1-methyl-2-pyrrolidinyl) pyridine, consists of a pyridine and a pyrrolidine ring, is volatile and has a molecular weight of 162.2. The concentration of nicotine in ECs in most cases ranges from $0 \mathrm{mg} / \mathrm{ml}$ to $36 \mathrm{mg} / \mathrm{ml}$. The amount of nicotine absorbed is affected by the device and the amount of e-liquids vaporized ${ }^{21}$. Smoking an ECs with a 30s interval for 10 times, the serum nicotine concentration increased significantly in $5 \mathrm{~min}$, indicating that nicotine in ECs is rapidly absorbed ${ }^{22}$. During smoking, nicotine is absorbed by the lungs and is rapidly metabolized in the liver, which induces three major adverse effects on the liver: toxic, immunological, and oncogenic effects ${ }^{23}$. The use of ECs may induce hepatic fibrosis, steatosis, cell dysfunction, injury with the elevation of liver enzymes and cancer ${ }^{24}$.

The toxic effect of ECs may be multifactorial. It is due to the increase in the formation of reactive oxygen species/oxidative stress, anti-inflammatory cytokines, DNA damage, hepatocyte apoptosis and necrosis, excess free fatty acids delivery (possibly through adipose tissue lipolysis), perturbations of cholesterol and lipid metabolism and epigenetic changes ${ }^{25}$. Oxidative stress is associated with many inflammatory diseases. It can cause membrane lipid rupture, protein denaturation, DNA damage, mitochondrial dysfunction and other cellular macro-molecular damage, thus severely altering signal transduction and cell metabolism. The use of ECs causes endothelial/ vascular dysfunction, nitric oxide deficiency. The use of ECs increases serum and hepatic iron which induces oxidative stress and lipid peroxidation that leads to activation of stellate cells and development of fibrosis ${ }^{26}$. On the other hand, nicotine-free e-liquid can cause depression in the activity of antioxidant enzymes which lead to increased oxidative stress. Oxidative stress seems to play a crucial role, and the Nrf2 (nuclear factor erythroid-2-related factor 2) signaling pathway plays a very important role in the process of oxidative stress ${ }^{27}$.

Some chemicals present in ECs (e.g., nicotine, formaldehyde, acrolein) are capable of causing DNA damage and mutagenesis. ECs induce hepatic DNA damage, decreasing levels of NAD+, elevated NADH levels and mitochondrial dysfunction ${ }^{28}$. Infiltration of inflammatory cells and cell death are also present. Nicotine increases the pro-inflammatory cytokines (IL-1, IL-6, IL-8 and tumor necrosis factor alpha) which are involved in liver cell injury ${ }^{29}$. Protein kinase $\mathrm{C}$ alpha signaling pathway appears to play an important role in the inflammatory response triggered by ECs containing nicotine ${ }^{30}$.

${ }^{18}$ Pessione F, Ramond MJ, Njapoum C, Duchatelle V, Degott C, et al. (2001) Cigarette smoking and hepatic lesions in patients with chronic hepatitis C. Hepatology 34(1):121-125.

${ }^{19}$ Lerner CA, Sundar IK, Yao H, Gerloff J, Ossip DJ (2015) Vapors produced by electronic cigarettes and e-juices with flavorings induce toxicity, oxidative stress, and inflammatory response in lung epithelial cells and in mouse lung. PLoS ONE 10(2): 1-26.

${ }^{20} \mathrm{Committee}$ on the review of the health effects systems.

${ }^{21}$ Theodore L Wagener and others (2017) Generation and third-generation electronic cigarette users. 26: 1-14.

${ }^{22}$ Vansickel AR, Eissenberg T (2013) Electronic cigarettes: effective nicotine delivery after acute administration. Nicotine and Tobacco Research 15(1): 267-270.

${ }^{23}$ Zayadi AR (2006) Heavy smoking and liver. World Journal of Gastroenterology 12(38): 6098-6101.

${ }^{24}$ Golli NE, Lamine AJ, Neffati H, Rahali D,Dallagi Y (2016) Impact of e-cigarette refill liquid with or without nicotine on liver function in adult rats. Toxicology Mechanisms and Methods 26(6): 419-426.

${ }^{25}$ Vansickel and Eissenberg.

${ }^{26}$ Carnevale R, Sciarretta S, Violi F, Nocella C, Loffredo L, et al. (2016) Acute impact of tobacco vs electronic cigarette smoking on oxidative stress and vascular function. Chest 150(3): 606-612.

${ }^{27}$ Cao and others.

${ }^{28}$ Derout JE, Shao XM, Bankole E, Hasan KM, Mtume N, et al. (2019) Hepatic DNA damage induced by electronic cigarette exposure is associated with the modulation of NAD+/PARP1/SIRT1 axis. Frontiers in Endocrinology 10: 1-9.

${ }^{29}$ El-Zayadi.

${ }^{30} \mathrm{Cao}$ and others. 
The immunologic effect of nicotine is the disturbance of both cellmediated and humoral immune responses. Nicotine decreases lymphocyte proliferation and differentiation including suppression of antibody-forming cells by inhibiting antigen-mediated signaling in T-cells and ribonucleotide reductase ${ }^{31}$. Furthermore, smoking induces apoptosis of lymphocytes by increasing the production of Fas (CD95) death receptor which permits them to be slaughtered by different cells communicating a surface protein called Fas ligand (FasL). Smoking induces decreased CD4+ cells, increased of CD8+ lymphocytes, impaired natural killer cell activity and increases the production of pro-inflammatory cytokines ${ }^{32}$.

Nicotine-containing ECs may alter the expression of genes related to cholesterol biosynthesis and lipid metabolism in liver. It can increase the level of free fatty acids by direct stimulation of lipolysis in adipocytes. These free fatty acids cause the accumulation of triglycerides in the liver ${ }^{33}$. Smoking has carcinogenic chemicals that increase the risk of hepatocellular carcinoma ${ }^{34}$. The use of ECs has been implicated in disruption of the normal pathways of cell cycle control, which may affect both immune competence and tumor progression. Smoking- induced fibrosis may be favor the development of hepatocellular carcinoma. Some chemicals present in e-cigarette aerosols (e.g., formaldehyde, acrolein) are capable of causing hepatic DNA damage and cancer ${ }^{35}$. Research data suggest that both the M1 and M2 subunits of ribonucleotide reductase participate in cellular functions that are important for determining malignant potential, and aberrant levels of ribonucleotide reductase expression and enzyme activity have been reported in human tumors $^{36}$. Nicotine has been associated with the suppression of p53 (tumor suppressor gene) ${ }^{37}$. Thus, ECs may provide a selective advantage to malignant cells by promoting tumor cell growth and suppressing the immune response to those cells ${ }^{38}$.

While evidence in humans for associations between ECs use and liver cancer is extremely sparse, more abundant data have been generated in the in vitro and in vivo settings, including some positive data and some negative data on mutagenesis of e-cigarette parts. Due to the mixed results across different experimental conditions and for different outcomes, clear, consistent signals have yet to be observed ${ }^{39}$. Despite the known negative consequences of tobacco smoking, numerous pregnant females keep on utilizing ECs dependent on their wellbeing insight as contrasted and tobacco. Nicotine can cross the placenta and has effects on fetal development. Therefore, during pregnancy smoking can result in multiple adverse consequences, including sudden infant death syndrome, and could result in altered corpus callosum, deficits in auditory processing, and obesity. Pregnant women must avoid ECs even if it is free of nicotine as it may be cause increase oxidative stress, induce inflammation that may be lead to multiple organs injury in mother and offspring ${ }^{40}$.

\section{Future Research}

Most of the studies included in this review were talking about the effect of nicotine on the liver. So, we recommend more studies in the future on the effect of other ECs components as propylene glycol, glycerol and flavorings. Also, we recommend more studies using ECs with zero or low concentration of nicotine because many studies in our review used a high concentration of nicotine. There is a need also to know the effect of ECs on humans as there is a lack of human studies. We recommended studying the effect of long-term exposure to ECs.

\section{Conclusion}

The biggest problem of ECs lies in the presence of nicotine in its composition as it causes many problems including liver toxicity, immunological effect and cancer. Pregnant women are advised to avoid ECs as their components may affect their newborns' liver.

\footnotetext{
${ }^{31}$ Jesica MC, Link KL, Eaton SS, Freed BM (2000) Exposure to cigarette tar inhibits ribonucleotide reductase and blocks lymphocyte proliferation. The Journal of Immunology 165(12): 6771-6775.

${ }^{32}$ El-Zayadi.

${ }^{33}$ El-Zayadi.

${ }^{34}$ El-Zayadi.

${ }^{35}$ Committee on the Review of the Health Effects Systems.

${ }^{36} \mathrm{McCue}$ and others.

${ }^{37}$ El-Zayadi.

${ }^{38} \mathrm{McCue}$ and others.

${ }^{39}$ Committee on the Review of the Health Effects Systems.

${ }^{40}$ Gerard Li, Chan YL, Wang B, Saad S, George J, et al. (2020) E-cigarettes damage the liver and alter nutrient metabolism in pregnant mice and their offspring. Annals of the New York Academy of Sciences 1475(1): 64-77.
} 


\section{References}

1. Priscilla CL (2014) Electronic Cigarettes: Human Health Effects. Tobacco Control 23(SUPPL 2).

2. Yuna C, Wu D, Ma Y, Ma X, Wang S, et al. (2021) Toxicity of electronic cigarettes: a general review of the origins, health hazards, and toxicity mechanisms. Science of the Total Environment 772: 145475.

3. Roberto C, Sciarretta S, Violi F, Nocella C, Loffredo L, et al. (2016) Acute impact of tobacco vs electronic cigarette smoking on oxidative stress and vascular function. Chest 150(3): 606-612.

4. (2018) Committee on the review of the health effects systems, Electronic nicotine delivery, National academies of sciences, engineering, and medicine; Health and medicine division; Board on population health and public health practice. In: Eaton DL, et al. (Eds.), (13 ${ }^{\text {th }}$ edn), WashingtonUnited States of America: Public health consequences of E-Cigarettes. National Academies Press, USA.

5. Zayadi E, Rahman A (2006) Heavy smoking and liver. World Journal of Gastroenterology 12(38): 6098-6101.

6. Derout JE, Shao XM, Bankole E, Hasan KM, Mtume N, et al. (2019) Hepatic DNA damage induced by electronic cigarette exposure is associated with the modulation of NAD+/PARP1/SIRT1 axis. Frontiers in Endocrinology 10: 1-9.

7. Golli NE, Lamine AJ, Neffati H, Rahali D, Dallagi Y, et al. (2016) Impact of E-cigarette refill liquid with or without nicotine on liver function in adult rats. Toxicology Mechanisms and Methods 26(6): 419-426.

8. Rachel G, Benowitz N, Glantz SA (2014) E-cigarettes a scientific review. Circulation 129(19): 1972-1986.

9. Gurjot K, Muthumalage T, Rahman I (2018) Mechanisms of toxicity and biomarkers of flavoring and flavor enhancing chemicals in emerging tobacco and non-tobacco products. Toxicology Letters 288: 143-155.

10. Chad AL, Sundar IK, Yao H, Gerloff J, Ossip DJ, et al. (2015) Vapors produced by electronic cigarettes and E-Juices with flavorings induce toxicity, oxidative stress, and inflammatory response in lung epithelial cells and in mouse lung. PLoS ONE 10(2): e0116732.

11. Gerard L, Chan YL, Wang B, Saad S, George J, et al. (2020) E-cigarettes damage the liver and alter nutrient metabolism in pregnant mice and their offspring. Annals of the New York Academy of Sciences 1475(1): 64-77.

12. Gerard L, Saad S, Oliver BG, Chen H (2018) Heat or burn? Impacts of intrauterine tobacco smoke and e-cigarette vapor exposure on the offspring's health outcome. Toxics 6(3): 43.

13. Majeed BA, Dube SR, Sterling K, Whitney C, Eriksen MP (2015) Opinions about electronic cigarette use in smoke-free areas among U.S. adults, 2012. Nicotine and Tobacco Research 17(6): 675-681.

14. Jesica MM, Link KL, Eaton SS, Freed BM (2000) Exposure to cigarette tar inhibits ribonucleotide reductase and blocks lymphocyte proliferation. The Journal of Immunology 165(12): 6771-6775

15. Vladimir BM, Brinkman MC, Granville CA, Gordon SM, Clark PI (2016) Original investigation real-time measurement of electronic cigarette aerosol size distribution and metals content analysis. Nicotine Tob Res 18(9): 1895-1902.

16. Fabienne P, Ramond MJ, Njapoum C, Duchatelle V, Degott C, et al. (2001) Cigarette smoking and hepatic lesions in patients with chronic hepatitis C. Hepatology 34(1):121-125.

17. Hanan Q Karim ZA, Rivera JO, Khasawneh FT, Alshbool FZ (2017) Impact of electronic cigarettes on the cardiovascular system. Journal of the American Heart Association 6(9): e006353.

18. (2010) U.S. Department of Health and Human Services, How tobacco smoke causes disease: The biology and behavioral basis for smokingattributable disease, How tobacco smoke causes disease: The biology and behavioral basis for smoking-Attributable disease: A Report of the Surgeon General.

19. Vansickel AR, Eissenberg T (2013) Electronic cigarettes: effective nicotine delivery after acute administration. Nicotine and Tobacco Research 15(1): 267-270.

20. Theodore LW, Floyd EL, Stepanov I, Driskill LM, Meier E, et al. (2017) Generation and third-generation electronic cigarette users. 26: 1-14.

21. Worsley DJ, Jones K, Marshman Z (2014) Patients are asking about e-cigarettes. What do we tell them? British Dental Journal 217(2): 91-95.

For possible submissions Click below: 